\title{
Epidermal Growth Factor based Therapy Promotes Intracellular Trafficking and Accumulation of its Receptor in the Nucleus of Fibroblasts from Diabetic Foot Ulcers
}

Viviana Falcón-Cama', Maday Fernández-Mayola', Yssel Mendoza-Marí1, Nelson Acosta-Rivero², Ariana García-Ojalvo', Ricardo Bringas-Pérez', Ivón Menéndez-Valdés', Mariuska Matos-Terrero', Lilianne López-Noudo', Rocío Garateix-Suárez', Karla Pereira-Yañez ${ }^{1}$, Maritza González ${ }^{3}$, Sirenia González-Pozos ${ }^{4}$, Juan Kourí-Flores ${ }^{4}$, William Savigne-Gutiérrez ${ }^{5}$, Ivonne Salgado ${ }^{5}$, Alejandro Hernández-Seara ${ }^{5}$, David G Armstrong ${ }^{6}$ and $^{4}$ Jorge Berlanga-Acosta ${ }^{1 *}$

${ }^{1}$ Center for Genetic Engineering and Biotechnology, Havana, Cuba

${ }^{2}$ National Center for Scientific Research, Havana, Cuba

${ }^{3}$ Latin American School of Medicine, Havana, Cuba

${ }^{4}$ Electronic Microscopy Unit, LaNSE, Mexico City, Mexico

${ }^{5}$ National Institute of Angiology and Vascular Surgery, Havana, Cuba

${ }^{6}$ Southern Arizona Limb Salvage Alliance (SALSA), Arizona Health Science Center: 1501 N, Campbell Ave. Arizona, USA

\begin{abstract}
Objective: To gain a better understanding of the Epidermal Growth Factor (EGF) Receptor (EGFR) activation, trafficking and biological response in diabetic foot ulcers (DFU), exposed to recombinant human EGF via intra-ulcer infiltration as a healing alternative.

Methods: We studied by immunoelectron microscopy the intracellular localization of the EGFR and Proliferating Cell Nuclear Antigen (PCNA) in fibroblast-like cells (FLC) from granulation tissue of DFU patients, collected before and at different time points after EGF treatment.

Results: EGF therapy appears to increase EGFR immunolabeling. At early time-points, EGFR labeling is observed predominantly in the nucleus, suggesting a fast EGFR internalization and nuclear translocation. Interestingly EGFR is also detected in the mitochondrial outer membrane. PCNA expression and trafficking were also detected in a time-dependent manner after EGF infiltration.

Conclusion: Differential subcellular distribution of EGFR and PCNA and accumulation in the nucleus, in a timepoint specific manner, supports the induction of an EGF-mediated activation program that is sustained for at least 24 hours after the EGF administration. These findings substantiate the therapeutic ability of EGF to restore the healing process in DFU.
\end{abstract}

Keywords: Diabetes; Diabetes complications; Diabetic foot ulcers; EGF; EGFR; PCNA; Immunoelectron microscopy

\section{Introduction}

Diabetic foot ulceration (DFU) is one of the most feared complications of diabetes, remaining as the universal cause of nontraumatic amputations resulting in significant disability, morbidity and mortality [1]. Diabetes-related ulcers and amputations are associated with high 5-year mortality rates that even surpass some aggressive forms of cancers [2]. Abnormal wound repair process of peripheral soft tissues has been proposed as ingredient to sustain and/or amplify diabetic ulcers [3]. Diabetes-associated healing impairment results from amalgamated systemic and local factors that converge to the establishment of a pro-senescent phenotype, along with mitogenic arrest and anticipated programmed cell death of granulation tissue fibroblasts [4]. Concurrently, keratinocytes, fibroblasts, myofibroblasts and endothelial precursor cells' migration, homing, proliferation and extracellular matrix (ECM) synthetic properties are impaired in diabetes [5].

Diabetic healing functional impairment has also been related to a substantial dysregulation in the availability and activity of growth factors [6]. Mounting evidences suggest that epidermal growth factor (EGF)/epidermal growth factor receptor (EGFR) system becomes deteriorated by diabetes [7] showing downregulation of EGFR tyrosine kinase activity in peripheral tissues [7]. EGF is perhaps the most broadly studied growth factor in relation to wound healing. It has been endowed with the biological competence for reverting the proliferative arrest that characterizes the chronic wounds phenotype [8]. On the other hand, locally prolonged bioavailability and timely receptor stimulation have been shown to be required for a significant EGFmediated impact in wound closure $[9,10]$. EGF binding to EGFR can drive receptor conformational changes, trans-auto-phosphorylation reactions, and ultimately the activation of different target genes involved in the most relevant events required for tissue repair [11]. Remarkably, the EGF/EGFR signaling axis has been considered as a particular target for decades in cancer research, developmental biology, and wound healing.

As an alternative to circumvent the hostile environment of diabetic wounds and to ensure an adequate EGF availability to its receptor in

${ }^{*}$ Corresponding author: Dr. Jorge Berlanga-Acosta, Center for Genetic Engineering and Biotechnology, Havana, Cuba, Tel: 537-250-44-79; Fax: 537-25074-79; E-mail: jorge.berlanga@cigb.edu.cu

Received July 23, 2016; Accepted August 05, 2016; Published August 10, 2016

Citation: Cama VF, Mayola MF, Marí YM, Rivero NA, Ojalvo AG, et al. (2016) Epidermal Growth Factor based Therapy Promotes Intracellular Trafficking and Accumulation of its Receptor in the Nucleus of Fibroblasts from Diabetic Foot Ulcers. J Diabetic Complications Med 1: 111.

Copyright: $\odot 2016$ Cama VF, et al. This is an open-access article distributed under the terms of the Creative Commons Attribution License, which permits unrestricted use, distribution, and reproduction in any medium, provided the original author and source are credited. 
responsive cells, our group has conducted the intra-ulcer infiltrations of EGF for 15 years [10,12-15]. Most recent data from about 3800 patients, confirmed treatment success in granulation, re-epithelialization and amputation reduction risk $(5 \%)$ relapses rates per year for Wagner's grade 3 to 5 wounds $[13,15]$.

Several studies have shown that, upon EGF stimulation, full-length EGFR translocates to the interior of the cell. Intracellular trafficking of EGFR involves the nucleus where it binds to c-myc (MYC) and cyclin D1 (CNND1) promoters, and phosphorylates the proliferating cell nuclear antigen (PCNA), all together committed in cell division. PCNA is important for both DNA synthesis and DNA repair, promoting division cell cycle progression from G1 to S phase [16]. Therefore, owing to its responsiveness to EGF and its commitment in cell proliferation, PCNA has been extensively used as a cell cycle progression marker [17]. However, little is known about EGFR cellular compartmentation and its biological response to natural ligands in vivo. Scarce information exists regarding the EGFR intracellular trafficking and the ensued downstream response in a human tissue exposed to EGF as a pharmacological agent in a clinical setting. Therefore, the goal of this study was to conduct an immunoelectron microscopy investigation to monitor, in a temporal sequence, the intracellular trafficking of both EGFR and PCNA in fibroblasts collected from DFU's samples of patients treated with locally infiltrated EGF.

\section{Methods}

\section{Ethics}

The study protocol conformed to the ethical guidelines of the 1975 Declaration of Helsinki. In addition, this protocol was reviewed and approved by the ethic committees at the National Institute of Angiology and Vascular Surgery, and the National Center for Integral Diabetes Care, Havana, Cuba. All the patients approved to be involved in the study and signed an informed consent.

\section{Study population}

Twelve diabetic patients (type 1 or 2 diabetes) affected by chronic neuropathic lower extremity wounds and admitted at the National Institute of Angiology and Vascular Surgery in Havana, Cuba, were included in this study. The wounds were classified as grades 3 and 4 according to the Wagner's scale [18-20]. All the patients were part of the National Program for Integral Diabetes Care. This program involves the intralesional infiltration of recombinant human EGF as instrumental adjunctive pharmacological intervention. Under the inhospital regime, the patients received the standard wound care including systemic medical interventions required to eliminate infection, proper metabolic control and the concurrent limb off-loading. Locally, wounds were cleansed with saline, sharp debrided when required, and dressed with saline-moistened gauze [21]. Wounds were clinically examined on alternate days and before EGF administration, as to confirm a clean wound substrate for infiltrations [22]. Blood glucose measurements were frequently indicated as for patients' metabolic control. The population study characterization is shown in Table 1.

\section{Intralesional infiltration with recombinant human EGF}

Recombinant human EGF was obtained from the Centre for Genetic Engineering and Biotechnology (Havana, Cuba) [23]. It was formulated with buffer salts as a lyophilized injectable, and is a commercially available composition (Heberprot-P, HeberBiotec S.A., Havana, Cuba) [24]. The ordinary protocol conceives $75 \mu \mathrm{g}$ of EGF/5 $\mathrm{mL}$ of saline solution at each intralesional infiltration session, three times per week on alternate days. The medication, its administration requisites and the procedure have been extensively described along progressive clinical trial publications $[13,15]$.

\section{Samples collection scheme}

Biopsy samples were harvested using commercially and disposable $2 \mathrm{~mm} \varnothing$ biotomes (Acuderm, USA). Time zero (T0) harvesting corresponds to the sample obtained just prior to the initial EGF infiltration, when the wound was fully preconditioned [25] and the metabolic status of the patient had been improved. This time window somewhat reflects the basal, constitutive expression of the EGFR in the ulcers' fibroblast cells and is used a reference point versus the subsequent sampling points data. All the patients were infiltrated with EGF simultaneously. Subsequent biopsy sampling time points were: 15 (T15), 45 (T45), and 60 (T60) minutes, and 6 (T6) and 24 (T24) hours following EGF infiltration. Granulation tissue fragments obtained at T0 and $\mathrm{T} 15$ were collected from the same four patients. In order to reduce patients' invasiveness, granulation tissue samples were subsequently collected from two different patients at each time point. All the wound bed and the dermo-epidermal contours were EGF-infiltrated and the samples were collected from a previously identified well-granulated corner $[13,15,25]$

\section{Transmission electron microscopy (TEM)}

Sampling was performed as described elsewhere [26]. Granulation tissue samples were fixed and analyzed by transmission electron microscopy as previously described [27,28]. Briefly, $2 \mathrm{~mm}$ thick fragments of granulation tissue samples were fixed for $1 \mathrm{~h}$ at $4^{\circ} \mathrm{C}$ in $1 \%$ $(\mathrm{v} / \mathrm{v})$ glutaraldehyde and $4 \%(\mathrm{v} / \mathrm{v})$ paraformaldehyde, rinsed in $0.1 \mathrm{M}$ sodium cacodylate ( $\mathrm{pH} 7.4$ ), post-fixed for $1 \mathrm{~h}$ at $4^{\circ} \mathrm{C}$ in $1 \% \mathrm{O}_{\mathrm{s}} \mathrm{O}_{4}$ and dehydrated in increasing concentrations of ethanol. Ultrathin sections (400-500 ̊) made with an ultramicrotome (NOVA, LKB), were placed on 400 mesh grids, stained with saturated uranyl acetate and lead citrate and examined with a JEOL/JEM 1400 transmission electron microscope (JEOL, Japan).

\section{Antibodies}

EGFR immunostaining was performed using a mouse monoclonal IgG that targets the extracellular domain of EGFR with high affinity. It was developed by the Center for Molecular Immunology (Havana, Cuba) and marketed by CIMAB S.A., Havana, Cuba [29]. PCNA was detected using a commercially available rabbit polyclonal IgG antibody (ab15497) (Abcam, Cambridge, United Kingdom) that recognizes human PCNA.

\section{Immunoelectron microscopy (IEM)}

Samples of granulation tissue were fixed with $4 \%$ (v/v) paraformaldehyde containing $0.1 \% \quad(\mathrm{v} / \mathrm{v})$ glutaraldehyde in 0.1

\begin{tabular}{|c|c|}
\hline Age (mean \pm SD) & $58.7 \pm 5.2$ \\
\hline Diabetes classification & $\begin{array}{l}\text { T1-DM: } N=3 \\
\text { T2-DM: } N=9\end{array}$ \\
\hline DM evolution (years) (mean \pm SD) & $16.5 \pm 7.1$ \\
\hline Wound classification (Wagner's scale (from 0 to 5 ) & $\begin{array}{l}\text { Grade 3: } N=5 \\
\text { Grade 4: } N=7\end{array}$ \\
\hline $\begin{array}{l}\text { Wound evolution (days) } \\
\text { (mean } \pm \text { SD) }\end{array}$ & $51 \pm 15.8$ \\
\hline Fasting glycaemia at time zero $(\mathrm{mmol} / \mathrm{L})$ & $8.2 \pm 0.65$ \\
\hline $\mathrm{HbA1c}(\%)$ & $9.3 \pm 0.82$ \\
\hline
\end{tabular}

Table 1: Demographic characteristic of the study population. 
Citation: Cama VF, Mayola MF, Marí YM, Rivero NA, Ojalvo AG, et al. (2016) Epidermal Growth Factor based Therapy Promotes Intracellular Trafficking and Accumulation of its Receptor in the Nucleus of Fibroblasts from Diabetic Foot Ulcers. J Diabetic Complications Med 1: 111.

Page 3 of 8

M phosphate buffer ( $\mathrm{pH} 7.3)$ at $4^{\circ} \mathrm{C}$ for $3 \mathrm{~h}$ and washed with $0.1 \mathrm{M}$ phosphate buffer, $\mathrm{pH}$ 7.3. Fixed samples were dehydrated as described above, embedded in Lowicryl, and polymerized by exposure to ultraviolet light at room temperature (RT) for $72 \mathrm{~h}$. Ultrathin sections of biopsies were incubated with either anti-EGFR or anti-PCNA antibodies in phosphate buffer, for $45 \mathrm{~min}$ at RT. The sections were rinsed three times for $30 \mathrm{~min}$ at RT with $0.1 \%$ bovine serum albumin in phosphate-buffered saline, $\mathrm{pH} 7.3$ (BSA-PBS), and incubated for 1 $\mathrm{h}$ at RT with gold-labeled (15 nm) anti-mouse IgG or anti-rabbit IgG (GE Healthcare Life Sciences, Mississauga, Ontario, Canada) diluted 1:100 in BSA-PBS. As internal immunolabeling control, the primary antibody was replaced by either normal mouse or rabbit serum. All sections were stained and analyzed with a transmission electron microscope, as mentioned above. At least ten photographs were obtained for each time-point and patient. The images were blindly and independently analyzed by two investigators (VFC and SG), focusing on the granulation tissue fibroblast-like cells (FLCs) as the principal granulation tissue cell.

Comparing distributions of gold particles between different compartments within a cell was done as described [30]. The objective was to test whether the observed distribution of gold particles between compartments within a cell is a random event. If not, then some compartments must be preferentially labeled. Gold particles representing the 'observed gold particles' (No) and a superimposed lattice of test points ('Test points', P), were counted in each cell compartment (endoplasmic reticulum (ER) and Golgi complex (GC) (ER+GC), mitochondria, plasma membrane, rest of cytoplasm (RC) (included all residual compartments in cytoplasm not of individual interest) and nucleus) using the ImageJ 1.38 software (http://imagej. nih.gov/ij). Then, the 'expected gold particles' (Ne) (i.e. random distribution), the relative labelling index: RLI (No/Ne), and a twosample Chi squared $\left(\mathrm{X}^{2}\right)$ analysis were calculated as previously described to test the null hypothesis (of no difference between distributions or the observed distribution of gold particles is random) for $\mathrm{r}$ rows- 1 degrees of freedom [26]. If the observed and expected distributions were different, preferentially labeled compartments can be identified on the basis of satisfying two criteria. First, the RLI value must be $>1$ and, second, the corresponding partial $\mathrm{X}^{2}$ value must account for a substantial proportion of total $\mathrm{X}^{2}$ [30]. To compare the distribution of gold particles in different compartments of FLCs at different times after treatment with EGF, we used a contingency table analysis (n columns $\mathrm{x} r$ rows) as described [30]. We considered the null hypothesis as 'no difference of gold distribution between FLCs at different times after treatment.

\section{Analysis of the regulatory relationship between EGFR and PCNA from data of the Encyclopedia of DNA elements}

Information on ChIP-seq experiments from ENCODE projects was obtained from the UCSC Genome Browser ftp site (hgdownload.cse. ucsc.edu). The file wgEncodeRegTfbsClustered.bed, containing data on transcription factor (TF) binding sites (TFBS) was downloaded. This file contains information on 161 TFs. The data on TFBSs includes chromosomal start and end positions of clusters of sequenced reads as results of ChIP set experiments. From the same site was downloaded therefGene.txt.gz file containing information on gene chromosomal positions. The TFBSfile was scanned and those binding sites located in promoters regions were assigned to the corresponding genes. Promoters were chosen as the DNA segments $(-2000,-1)$ relative to the transcription start site.

\section{Results}

\section{Study population}

As illustrated in Table 1, most of our patients (75\%) suffered for more than 15 years of type 2 diabetes. The targeted lesions included clean, debrided ulcers and amputation residual bases which, given their slow trajectory and torpid granulation process, were clinically entitled as chronic wounds. Most of them were classified as complex wounds by involving not only soft tissues, but bone, capsules and tendons. Given the in-hospital regime of metabolic control and other medical interventions, the patients achieved an acceptable compensation state.

\section{EGF therapy increases EGFR immunolabeling and its subcellular trafficking}

Immunolabeling of EGFR in Fibroblast-like cells (FLC) of biopsies obtained from patients with diabetic foot ulcer. Samples were incubated with an anti-EGFR mouse monoclonal antibody. Figures depict a time sequence of activation and trafficking events upon local infiltration of EGF (Heberprot-P) within the ulcers (Figure 1). As shown in Figure 1A, EGFR was barely recognized on FLCs in samples obtained minutes prior to the initial EGF treatment (T0). Early after EGF therapy (from T15 to T60), increased anti-EGFR immunostaining was observed in the rough endoplasmic reticulum (RER), in nucleus and mitochondria of FLCs (Figure 1B, Table 2). Immunolabeling was also shown in various intracellular vesicles near the plasma membrane, RER and GC (Figure 1B). It is interesting to note that following EGF treatment, immunolabeling was seen in the extracellular matrix (ECM), in exosome-like structures (ELS) and on collagen-like fibers (CF).

At a later time points after EGF treatment (T6), EGFR was specially immunolabelled in RER, GC and mitochondria (Table 2). Immunostaining was also detected in nucleus, various vesicles, multivesicular bodies (MVBs) and phagosomes (Figure 1Cb). Besides, gold particles were seen in the ECM, in ELS and on CF (Figure 1Ca). EGFR immunolabeling was observed on plasma membrane in contact with $\mathrm{CF}$ (Figure 1Ca). A similar pattern of EGFR detection was observed in FLCs at T24 after EGF infiltration (Figure 1D). A

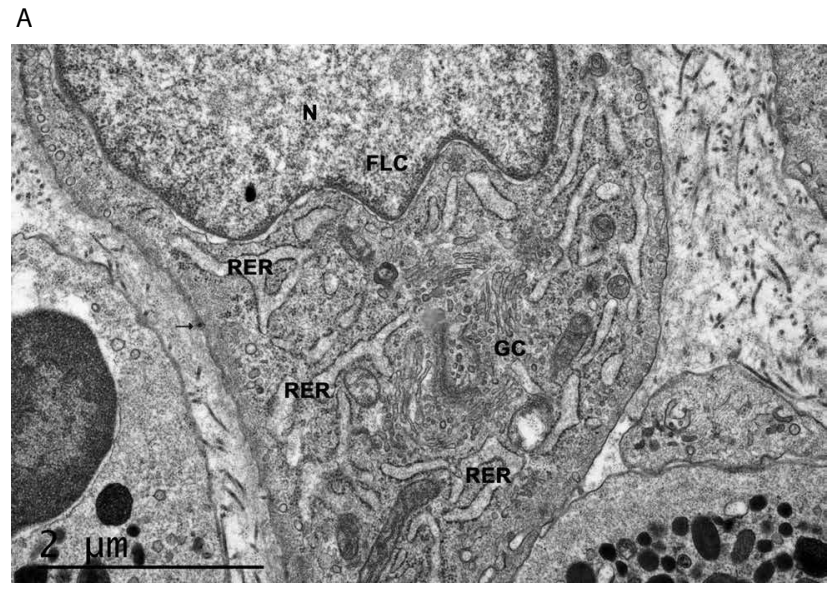

Figure 1A: Time Zero (T0) harvesting corresponds to the sample obtained minutes prior to the initial EGF infiltration. The image shows a negligible immunostaining on the plasma membrane of a Fibroblast-like cell (arrow). Rough endoplasmic reticulum (RER); Nucleus (N); Golgi complex (GC); fibroblast-like cell (FLC) (Bar=2 um). 
Citation: Cama VF, Mayola MF, Marí YM, Rivero NA, Ojalvo AG, et al. (2016) Epidermal Growth Factor based Therapy Promotes Intracellular Trafficking and Accumulation of its Receptor in the Nucleus of Fibroblasts from Diabetic Foot Ulcers. J Diabetic Complications Med 1: 111.

Page 4 of 8

B

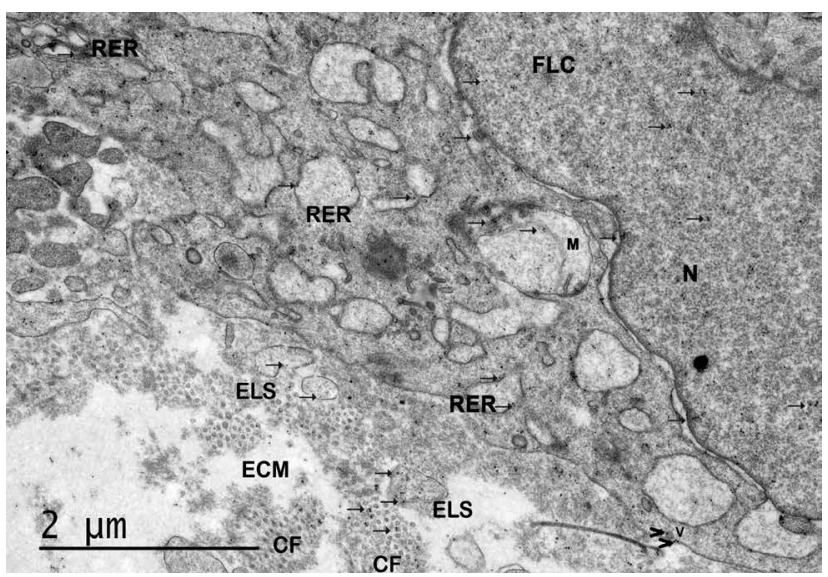

Figure 1B: Immunolabeling of EGFR (arrows) in part of a Fibroblast-like cell (FLC) from a biopsy sample obtained 45 minutes after EGF infiltration. Immunostaining appeared in mitochondria, RER and nucleus. Also note that EGFR was immunolabeled in vesicles (V) (arrowhead), on plasma membrane (arrowhead), the extracellular matrix (ECM), on collagen-like fibers (CF) and exosome-like structures (ELS) (Bar=2 um). preferential immunolabeling was shown in RER, and also in nucleus, mitochondria and vesicles. Interestingly, EGFR was seen on RER membranes adjacent to mitochondria.

To test whether the overall distribution of labeling of compartments was consistent with a random pattern within FLCs, we compared the RLI values for different compartments. As shown in Table 2, the distribution pattern of gold particles in different cell compartments was significantly different from random. In FLCs analyzed at early times after EGF treatment, a preferential labeling was shown in mitochondria $\left(\mathrm{RLI}=1.91\right.$ and $58.40 \%$ of the total $\mathrm{X}^{2}$ ). At later time points after EGF treatment (T6-T24), preferential gold labeling of $\mathrm{ER}+\mathrm{GC}(\mathrm{RLI}=1.24$ and $30.07 \%$ of the total $\mathrm{X}^{2}$ ) and mitochondria (RLI $=1.60$ and $42.86 \%$ of the total $\mathrm{X}^{2}$ ) were observed (Table 2).

Gold labeling over the same compartments of FLCs obtained either at early or at late time points after treatment with EGF were also compared (Table 3). This analysis indicated that the distribution of EGFR labeling in FLCs analyzed at different times is significantly different. Data indicated that the major contributors to the difference were the nucleus $\left(40.73 \%\right.$ of the total $\left.\mathrm{X}^{2}\right)$, mitochondria $(40.07 \%$ of the total $\left.\mathrm{X}^{2}\right)$ and $\mathrm{ER}+\mathrm{GC}\left(15.25 \%\right.$ of the total $\left.\mathrm{X}^{2}\right)$.

\begin{tabular}{|c|c|c|c|c|c|c|c|c|c|c|c|c|}
\hline \multirow[t]{2}{*}{ Compartments } & \multicolumn{3}{|c|}{ EGFR (T15-T60) } & \multicolumn{3}{|c|}{ EGFR (T6-T24) } & \multicolumn{3}{|c|}{ PCNA (T15-T60) } & \multicolumn{3}{|c|}{ PCNA (T6-T24) } \\
\hline & RLI & $\mathbf{X}^{2}$ & $\mathrm{X}^{2} \%$ & RLI & $\mathbf{X}^{2}$ & $X^{2} \%$ & RLI & $\mathbf{X}^{2}$ & $\mathrm{X}^{2} \%$ & RLI & $\mathbf{X}^{2}$ & $X^{2} \%$ \\
\hline$E R+G C$ & 1.09 & 1.44 & 7.44 & 1.24 & 15.04 & 30.07 & 1.35 & 16.81 & 63.71 & 1.16 & 3.41 & 12.42 \\
\hline$M$ & 1.91 & 11.29 & 58.40 & 1.60 & 21.44 & 42.86 & 1.31 & 2.53 & 9.59 & 1.36 & 7.41 & 27.00 \\
\hline $\mathrm{RC}$ & 0.89 & 5.00 & 25.86 & 0.91 & 3.84 & 7.68 & 0.86 & 6.02 & 22.80 & 1.05 & 0.69 & 2.52 \\
\hline PM & 0.90 & 0.22 & 1.12 & 0.70 & 2.30 & 4.60 & 1.15 & 0.34 & 1.29 & 1.01 & 0.00 & 0.02 \\
\hline $\mathrm{N}$ & 1.08 & 1.39 & 7.19 & 0.84 & 7.40 & 14.80 & 0.95 & 0.69 & 2.62 & 0.72 & 15.93 & 58.05 \\
\hline Total & & 19.33 & 100 & & 50.02 & 100 & & 26.39 & & & 27.44 & \\
\hline
\end{tabular}

For degrees of freedom $(\mathrm{df})=4$ (2-1 columns by 5-1 rows), and total chi-squared values of: (EGFR) T15-T60: $\mathbf{X}^{2}=19.33, \mathrm{P}<0.001 ; \mathrm{T} 6-\mathrm{T} 24: \mathbf{X}^{2}=50.02$, P<0.001; (PCNA) T15-T60: $\mathbf{X}^{2}=26.39, P<0.001$; T45-T60: $\mathbf{X}^{2}=27.44, \mathrm{P}<0.001$; so the distribution patterns of gold particles is significantly different from random. For EGFR, at T15-T60, mitochondria (RLI=1.91 and $58.40 \%$ of the total) meet the two criteria for preferential labelling: 1) RLI is greater than 1 , and 2 ) partial $\mathbf{X}^{2}$ values make substantial contributions to the total, $>10 \%$. At T6-T24, there is preferential labelling of ER+GC (RLI=1.24 and $30.07 \%$ of the total) and mitochondria (RLI=1.60 and $42.86 \%$ of the total). For PCNA, at T15-T60, there is preferential labeling of ER+GC (RLI=1.35 and $63.71 \%$ of the total). At T6-24, there is preferential labeling of ER+GC (RLI=1.16 and $12.42 \%$ of the total) and mitochondria (RLI=1.36 and $27.00 \%$ of the total). RLI, Relative Labeling Index; $X^{2}$, chi-squared values; $X^{2} \%$, chi-squared values expressed as percent; ER: Endoplasmic Reticulum; GC: Golgi Complex; M: Mitochondria; RC: Rest Of Cytoplasm; PM: Plasma Membrane; N; Nucleus.

Table 2: Relative labeling index (RLI) for EGFR and PCNA labeled by gold particles in different compartments of fibroblast-like cells (FLCs), analyzed at early (from T15 to T60) or later time-points (from T6 to T24) after EGF treatment.
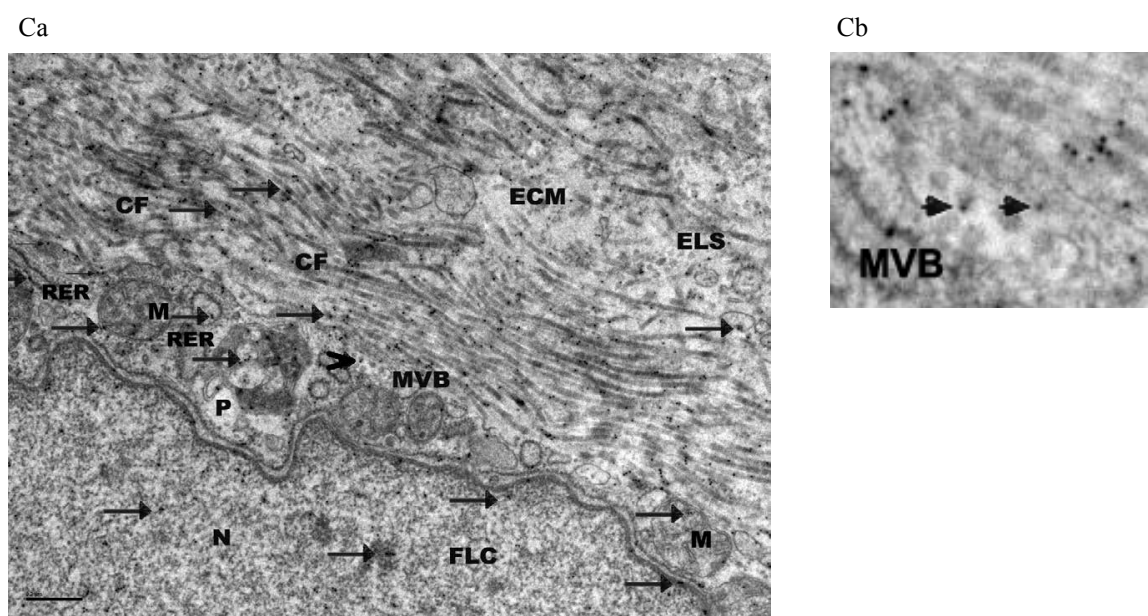

Figure 1C: a: Immunolabeling of EGFR (arrows) in part of a Fibroblast-like cell (FLC) and extracellular matrix (ECM) from a sample obtained 6 hours after EGF infiltration (T6) of a diabetic foot ulcer. Note immunolabeling of a multivesicular body (MVB) (arrowhead), phagosome (P), rough endoplasmic reticulum (RER), mitochondria (M), nucleus (N), collagen-like fibers (CF) and exosome-like structures (ELS). Also note immunostaining of plasma membrane (PM) and CF in contact with PM (small arrows). (Bar=2 um). C) b: Enlargement of a MVB showing EGFR immunolabeling (arrowheads). 
Citation: Cama VF, Mayola MF, Marí YM, Rivero NA, Ojalvo AG, et al. (2016) Epidermal Growth Factor based Therapy Promotes Intracellular Trafficking and Accumulation of its Receptor in the Nucleus of Fibroblasts from Diabetic Foot Ulcers. J Diabetic Complications Med 1: 111.

Page 5 of 8

$\mathrm{D}$

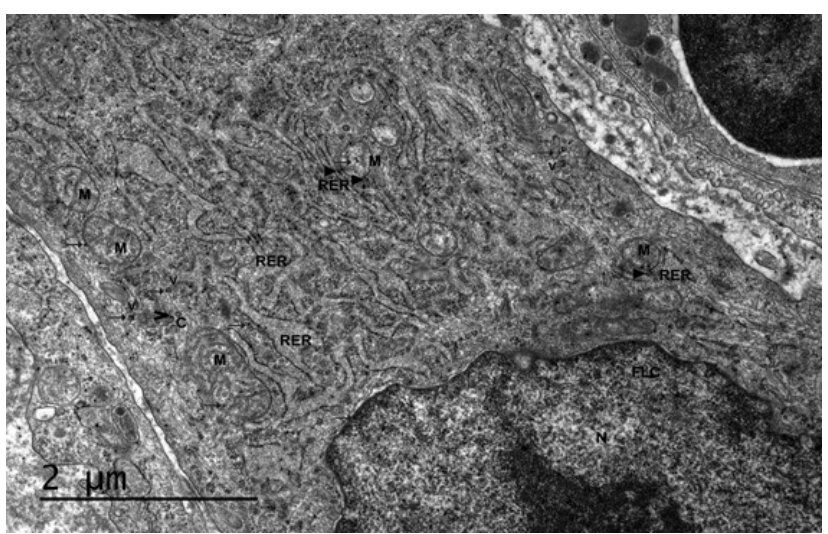

Figure 1D: This image corresponds to EGFR immunolabeling (arrows) in a Fibroblast-like cell (FLC) from a diabetic foot ulcer sample, 24 hours (T24) after treatment with EGF. Preferential immunolabeling was observed in RER. In addition, EGFR was detected in nucleus (N), mitochondria (M) and vesicles $(V)$. Note immunolabeling on membranes of RER in contact with mitochondria (filled arrowhead) and in a caveosome-like structure (C) (arrowhead) (Bar=0.2 um).

A

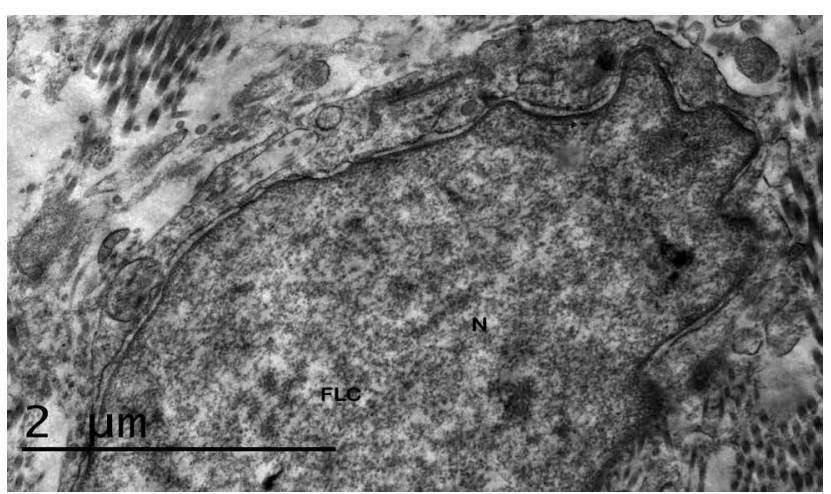

Figure 2A: The image corresponding to T0, prior to the first EGF infiltration, shows scarce PCNA immunostaining in the nucleus $(\mathrm{N})$ region. (Bar=2 um).

\section{EGF therapy increases PCNA immunolabeling and its subcellular trafficking}

Immunolabeling of PCNA, as a downstream target of nuclear EGFR kinase activity as well as a DNA replication and cell proliferation marker. Samples were incubated with anti-PCNA rabbit polyclonal antibodies. Figures depict a time sequence of activation and localization events upon local infiltration of EGF (Heberprot-P) in Fibroblastlike cells (FLCs) within the ulcers (Figure 2). Prior to EGF injection (T0), PCNA expression was scarcely detected in FLCs (Figure 2A). Remarkably, at early times following exposure to EGF, increased immunolabeling was observed in nucleus, $\mathrm{ER}+\mathrm{GC}$ and mitochondria (Figure 2B, Table 2). PCNA was also detected on RER membranes adjacent to mitochondria, on plasma membrane and in MVBs (Figure 2B). Similarly, at later time points after EGF treatment (T6-T24), PCNA appeared particularly immunolabelled in $\mathrm{ER}+\mathrm{GC}$ and mitochondria (Figures 2C and 2D). In addition, PCNA immunostaining was shown in nucleus. The distribution pattern of gold particles in different cell compartments within FLCs was significantly different from random (Table 2). In FLCs analyzed at T15-T60, a special PCNA labeling was seen in $\mathrm{ER}+\mathrm{GC}$ (RLI=1.35 and $63.71 \%$ of the total). At T6-T24 after EGF treatment, gold particles were also preferentially found in ER+GC (RLI $=1.16$ and $12.42 \%$ of the total) and mitochondria (RLI $=1.36$ and $27.00 \%$ of the total) (Table 2). Comparison of gold labeling over the same compartments of FLCs at different time points indicated that there were significant differences among the distributions of labeling in these groups (Table 3). Compartmental $\mathrm{X}^{2}$ values indicate that the major contributors to the difference were the nucleus $(47.13 \%$ of the total $\left.\mathrm{X}^{2}\right)$, mitochondria $\left(35.2 \%\right.$ of the total $\left.\mathrm{X}^{2}\right)$ and $\mathrm{RC}(14.81 \%$ of the total $\mathrm{X}^{2}$ ).

\section{Analysis of the regulatory relationship between EGFR and PCNA from data of the Encyclopedia of DNA Elements}

With the aim of gaining further information on the regulatory relationship between EGFR and PCNA, we analyzed data from the

B

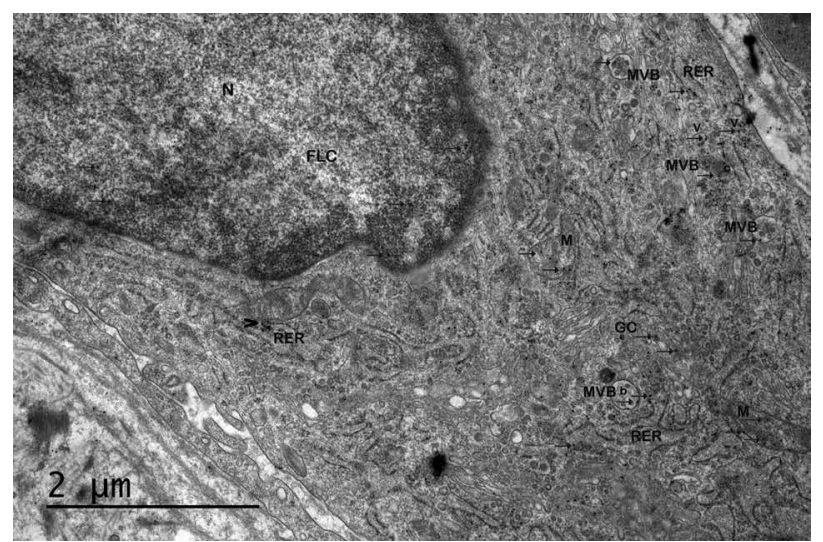

Figure 2B: Immunolabeling of PCNA in a Fibroblast-like cell (FLC) from a biopsy of samples infiltrated with EGF at T60. PCNA labeling mostly accumulated in RER and Golgi complex (GC). Immunolabeling was also detected on the plasma membrane, in the nuclear region $(\mathrm{N})$, mitochondria (M), and multivesicular bodies (MVBs). Note that PCNA was detected on RER membranes adjacent to mitochondria (arrowhead) (Bar=2 um).

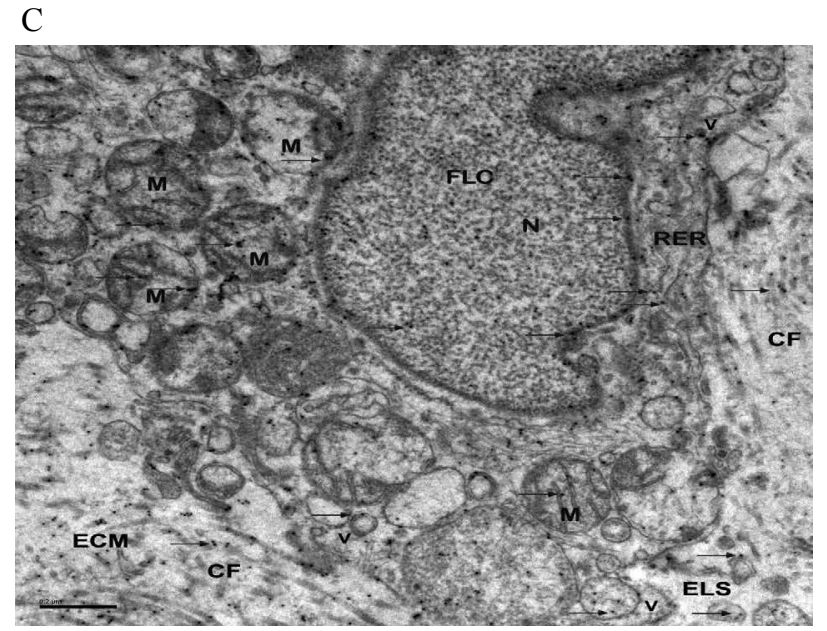

Figure 2C: Immunolabeling of PCNA (arrows) in part of a fibroblast-like cell (FLC) from a biopsy taken at T6. PCNA immunostaining was shown in mitochondria (M), RER and nucleus (N). Also note immunolabeling in vesicles $(\mathrm{V})$ and in the ECM on collagen-like fibers (CF) and exosome-like structures (ELS). (Bar=0.2 um). 
Citation: Cama VF, Mayola MF, Marí YM, Rivero NA, Ojalvo AG, et al. (2016) Epidermal Growth Factor based Therapy Promotes Intracellular Trafficking and Accumulation of its Receptor in the Nucleus of Fibroblasts from Diabetic Foot Ulcers. J Diabetic Complications Med 1: 111.

Page 6 of 8

$\mathrm{D}$

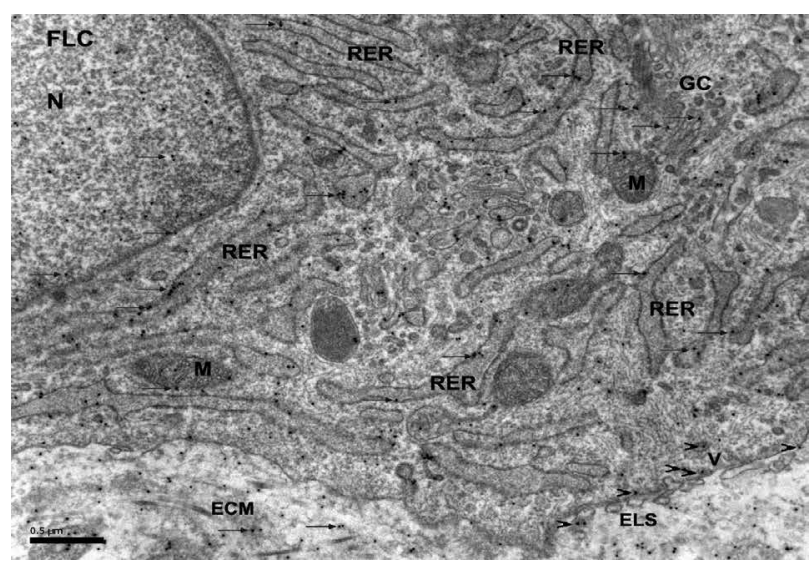

Figure 2D: Immunolabeling of PCNA in part of a fibroblast-like cell (FLC) from a biopsy of samples infiltrated with EGF at T24. Large labeling of RER, Golgi complex (GC) and mitochondria (M) were observed. Immunostaining of PCNA was also detected in nucleus $(\mathrm{N})$, and extracellular matrix (ECM) (arrows). Also note immunostaining in plasma membrane, vesicles and exosome-like structures (ELS) (arrowheads) (Bar=0.5um)

Encyclopedia of DNA Elements (ENCODE project). TF-interactions were obtained as described in Materials and Methods section. Processed data included information for a total of $161 \mathrm{TFs}$. We identified a total of 11 TFs (ATF3, E2F1, MAX, MYC, NR3C1, POLR2A, RAD21, RBBP5, SIN3A, TAF1 and ZNF263) that bind to both, EGFR and PCNA promoter regions. Additionally we found three transcription factors, MYC, ELK1 and STAT5 that are activated in the EGF/EGFR signaling pathway and were found to bind PCNA gene promoter according to ChIP Seed data (Figure 3).

\section{Discussion}

Despite the present study has the merit to provide the first evidences on the EGFR and PCNA intracellular kinetic in response to a therapeutic intervention with EGFR ligand in a clinical scenario; the findings impact is shadowed by not having a parallel, concurrent control group with a similar morbidity pattern in which EGF infiltration would have been replaced with a placebo solution. This was due to ethical reasons. Therefore, all the data presented here are based on a comparison of the different and progressive time-point samplings versus $\mathrm{T} 0$ in the same patient.

Activation of the EGFR has been shown to be critical in wound healing $[31,32]$. Within the context of diabetic chronic wounds, a sort of "tyrosine kinase paralysis" and EGFR functional deficiency has been demonstrated in fibroblasts exposed to a hostile glycation environment [7]. Thus, in diabetes-associated healing impairment, the EGF/EGFR system has been shown to be affected because of low levels of EGF and EGFR cell signaling downregulation [7,33]. According to our data, EGFR is also scarcely detected in the fibroblasts derived from diabetic granulation tissue before receiving EGF treatment. This, in theory, may represent an additional factor contributing to the deterioration of signaling through this receptor pathway.

Although one of the limitations of this work is that T0 is the only reference control, the study suggests that intralesional EGF therapy induced the activation of EGFR in FLCs early after EGF administration (from T15 to T60). First, there was an increase of EGFR immunolabeling upon EGF exposure. Second, early after EGF treatment, accumulation of EGFR labeling was observed in the nucleus and to a lesser extent in other cytoplasmic compartments, suggesting EGFR internalization and nuclear translocation. Moreover, EGFR differential subcellular distribution and accumulation in the ECM, in a time-point manner, support the induction of an EGF-mediated activation program that is sustained for at least $24 \mathrm{~h}$. Finally, detection of EGFR was related to a parallel immunolabeling increase and differential subcellular distribution of its downstream signaling target, PCNA. Accordingly, both EGFR and PCNA were particularly immunolabelled in the nucleus early after EGF treatment. These results converge with previous reports in cell culture systems showing that EGF increases EGFR gene and protein expression, and inhibits both EGFR mRNA decay and degradation of internalized EGFR by lysosomes and proteasome [34].

Comparison of different cellular compartments at several time points following EGF therapy indicates a shift in gold labeling of EGFR early after EGF therapy, with fewer-than-expected tags in $\mathrm{ER}+\mathrm{GC}$ and mitochondria, but more-than-expected in nucleus (Table 3). This suggests internalization and nuclear translocation of EGFR. Interestingly, at later time points after EGF treatment (T6-T24), a shift in labeling showed more-than-expected gold particles in ER+GC

\begin{tabular}{|c|c|c|c|c|c|c|c|c|c|c|c|c|c|c|}
\hline \multirow[t]{2}{*}{ Compartment } & \multicolumn{2}{|c|}{$\begin{array}{c}\text { EGFR } \\
\text { (T15-T60) }\end{array}$} & \multicolumn{2}{|c|}{$\begin{array}{c}\text { EGFR } \\
\text { (T6-T24) }\end{array}$} & \multirow[t]{2}{*}{$\begin{array}{l}\text { Row } \\
\text { total }\end{array}$} & \multirow{2}{*}{$\begin{array}{c}\text { EGFR } \\
\text { (T15-T60) } \\
X^{2}\end{array}$} & \multirow{2}{*}{$\begin{array}{c}\text { EGFR } \\
\text { (T6-T24) } \\
\mathbf{X}^{2}\end{array}$} & \multirow{2}{*}{$\begin{array}{c}\text { PCNA } \\
\text { (T15-T60) } \\
\text { No }\end{array}$} & \multirow{2}{*}{$\begin{array}{l}\text { PCNA } \\
\text { (T6-T24) } \\
\text { Expected } \\
\text { Golds }\end{array}$} & \multirow{2}{*}{\begin{tabular}{|c|}
$\begin{array}{l}\text { Row } \\
\text { total }\end{array}$ \\
No
\end{tabular}} & \multirow{2}{*}{$\begin{array}{c}\text { PCNA } \\
\text { T15-T60 } \\
\text { Expected } \\
\text { Golds }\end{array}$} & \multirow[t]{2}{*}{$\begin{array}{l}\text { PCNA } \\
\text { T6-T24 }\end{array}$} & \multirow{2}{*}{$\begin{array}{c}\text { PCNA } \\
\text { T15-T60 } \\
\mathrm{X}^{2}\end{array}$} & \multirow{2}{*}{$\begin{array}{c}\text { PCNA } \\
\text { T6-T24 } \\
X^{2}\end{array}$} \\
\hline & No & $\begin{array}{l}\text { Expected } \\
\text { Golds }\end{array}$ & No & $\begin{array}{l}\text { Expected } \\
\text { Golds }\end{array}$ & & & & & & & & & & \\
\hline $\mathrm{ER}+\mathrm{GC}$ & 178 & 209.2 & 315 & 283.8 & 493 & 4.64 & 3.4 & 184 & 174.6 & 148 & 157.4 & 332 & 0.5 & 0.6 \\
\hline M & 26 & 50.9 & 94 & 69.1 & 120 & 12.2 & 9.0 & 35 & 59.4 & 78 & 53.6 & 113 & 10.0 & 11.1 \\
\hline $\mathrm{RC}$ & 340 & 339.4 & 460 & 460.6 & 800 & 0.00 & 0.00 & 282 & 318.7 & 324 & 287.3 & 606 & 4.2 & 4.7 \\
\hline PM & 21 & 16.5 & 18 & 22.45 & 39 & 1.2 & 0.9 & 18 & 20.5 & 21 & 18.5 & 39 & 0.3 & 0.3 \\
\hline $\mathrm{N}$ & 261 & 210.0 & 234 & 285.0 & 495 & 12.4 & 9.1 & 273 & 218.8 & 143 & 197.2 & 416 & 13.4 & 14.9 \\
\hline Total & 826 & 826 & 1121 & 1121 & 1947 & & 52.8 & 792 & 792 & 714 & 714 & 1506 & & 60.2 \\
\hline
\end{tabular}

For degrees of freedom $(\mathrm{df})=4(2-1$ columns by $5-1$ rows $)$ and total chi-squared values for $E G F R,\left(X^{2}\right)=52.82, P<0.0001$; and $P C N A,\left(X^{2}\right)=60.15, P<0.0001$ (contingency table analysis). So, the null hypothesis (no difference between labelling distributions obtained at different time points) must be rejected. Compartmental $X^{2}$ values for EGFR indicate that the major contributors to the difference were the nucleus $\left(40.73 \%\right.$ of the total $\left.X^{2}\right)$, mitochondria $\left(40.07 \%\right.$ of the total $\left.X^{2}\right)$ and $E R+G C\left(15.25 \%\right.$ of the total $\left.X^{2}\right)$. Note that FLCs analyzed at T15-T60 have fewer-than-expected gold particles in both ER+GC and mitochondria while FLCs analyzed at T6-T24 have more-than-expected gold particles in both ER+GC and mitochondria. Also note that FLCs at T15-T60 showed more-than-expected gold particles in nucleus while FLCs analyzed at T6-T24 showed fewer-than-expected gold particles in nucleus. Compartmental $X^{2}$ values for PCNA indicate that the major contributors to the difference are nucleus (47.13\% of the total), mitochondria (35.2\% of the total) and RC (14.81\%). Note that FLCs analyzed at T15-T60 have fewer-than-expected gold particles in mitochondria and RC while FLCs analyzed at T6-T24 have more-than-expected gold particles in mitochondria and RC. Also note that FLCs at T15-T60 showed more-than-expected gold particles in nucleus while FLCs analyzed at T6-T24 showed fewer-than-expected gold particles in nucleus. No: Observed gold particles; P: Test Points; LD: Labelling Density; ER: Endoplasmic Reticulum; GC: Golgi complex); M: Mitochondria; RC: Rest of Cytoplasm; PM: Plasma membrane; N: Nucleus.

Table 3: Observed and expected distributions of gold particles for EGFR and PCNA in organelle compartments of fibroblast-like cells (FLCs) obtained either at early (from T15 to T60) or later time-points (from T6 to T24) after treatment with EGF and calculation of chi-squared ( $\left.\mathbf{X}^{2}\right)$ values in contingency table analysis. 


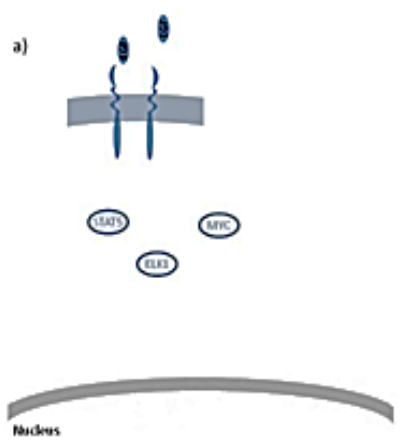

b)
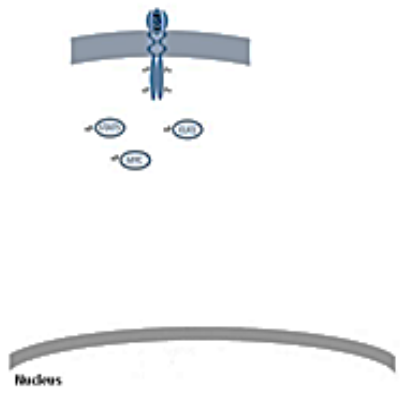
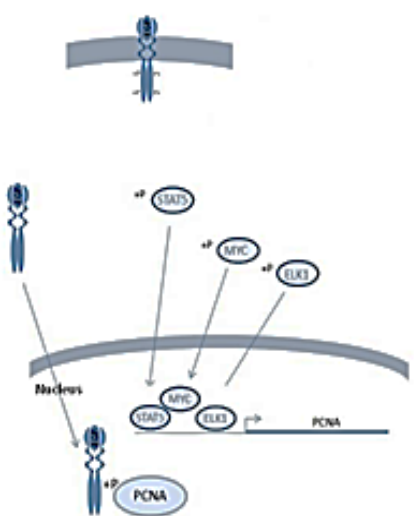

Figure 3: EGF delivery (a) induces the dimerization and autophosphorylation (b) of the EGFR cytoplasmic domain. Next, downstream signaling is activated by the binding of several proteins to EGFR phosphotyrosines and internalized. As a result of early EGF-EGFR signaling, three of the transcription factors activated by EGFR (STAT5, ELK1 and MYC) translocates to the nucleus. In the nucleus, they bind the PCNA promoter to induce early PCNA transcription. Later on, EGFR also accumulates in nucleus and phosphorylates PCNA promoting increased cell proliferation.

and mitochondria, but fewer-than-expected in nucleus, suggesting recycling of EGFR from nucleus to cytoplasm compartments and an increased trafficking of EGFR through the secretory pathway. In addition, detection of EGFR in various vesicles, endocytic vesicles, caveosomes-like structures, MVBs as well as phagosomes, supports the intracellular trafficking of the EGFR. These observations are in line with those derived from in vitro studies documenting an EGFR retrograde trafficking [35].

Previously, the EGF infiltration treatment has been proved to increase local EGF concentration in compromised tissues, promoting cytoprotection and cells proliferation [10]. Thus, EGF pharmacological concentration and EGFR levels in fibroblasts might possibly sustain increased receptor signaling (at least for $24 \mathrm{~h}$ after EGF treatment), cellular activation and the wound healing-related response observed in treated populations.

Results from this work showed EGFR immunolabeling as an exosome-related product. Several biological functions have been described for exosomes as mediators in intercellular signal communication by delivering proteins, lipids, mRNAs, microRNAs, and DNAs [36]. Thus, our finding presupposes that the accumulation of EGFR and EGFR-containing exosomes-like vesicles in the ECM might be potentially relevant. It would be interesting to study the function of these structures, as exosomes derived from mesenchymal stem cells have been shown to facilitate cutaneous wound healing [37,38]. Identification of EGFR in mitochondria of FLCs is another valuable finding of this work. Previous in vitro studies have found that EGFR localization to mitochondria is related to cell survival $[39,40]$.

It is relevant to note that EGFR preferentially accumulated in the nucleus of FLCs early after EGF therapy. These results are in line with previous evidences from in vitro models showing that full length EGFR translocates to the cell nucleus after ligand binding [16,41]. Several functions of nuclear EGFR have been described. Firstly, it has been shown to operate as a co-transcription factor regulating the expression of various genes, including cyclin D1 [42]. Moreover, EGFR has been demonstrated to interact with DNA-dependent protein kinase (DNAPK), leading to repair DNA double strand break [43]. Furthermore, nuclear EGFR has been described to phosphorylate chromatinbound PCNA, thus increasing PCNA stability and enhancing cellular proliferation $[41,42]$. Nuclear localization of EGFR is consistent with the findings of this study, derived from the PCNA expression and intracellular trafficking in fibroblasts upon EGF infiltration.

The analysis of TF-gene regulatory interactions from the ENCODE project identified three of the transcription factors activated by the early EGF-EGFR signaling (STAT5, ELK1 and MYC) that bind the PCNA promoter to induce PCNA transcription. This is particularly significant since PCNA has been involved in many critical cellular functions including cell cycle control, chromatin remodeling, gene expression and apoptosis [44]. It is worth mentioning that the observed increase of PCNA immunolabeling and its subcellular distribution was related with EGFR nuclear accumulation. These results suggest that the therapeutic effect of EGF treatment on diabetic ulcers healing may be partially related to EGFR-mediated PCNA functions in the nucleus of fibroblasts.

A change in intracellular localization, from nucleus to cytoplasm, has also been observed for PCNA but not for other nuclear proteins in neutrophils and fibroblasts after serum starvation [45]. Interestingly, this intracellular PCNA localization was related to the anti-apoptotic activity of PCNA in neutrophils. Hence, the therapeutic effect of EGF might also be related to increased expression and the functions of cytoplasmic PCNA in fibroblasts from DFU.

Finally, activation of both EGFR and PCNA in our fibroblasts was detected up to 24 hours following EGF treatment, suggesting a sustained effect of this therapy. These data lend consistency to the current ulcerinfiltration protocol, based on every 48 hours scheme so as to its ensued therapeutic impact $[13,15]$. Conclusively, EGF infiltrative intervention appeared to increase the levels of EGFR in "dormant" wounds fibroblasts, so as its intracellular trafficking in a time-sequential manner. Furthermore, its activation and nuclear translocation suggest a role in activating PCNA and its ensued proliferative effect. Globally speaking, all these findings may theoretically account for the therapeutic ability of EGF to restore the healing process in DFU.

\section{References}

1. Tuttolomondo A, Maida C, Pinto A (2015) Diabetic foot syndrome as possible cardiovascular marker in diabetic patients. Journal of diabetes research 2015: 1-12.

2. Morbach S, Furchert H, Gröblinghoff U, Hoffmeier H, Kersten K, et al. (2012) Long-term prognosis of diabetic foot patients and their limbs: amputation and death over the course of a decade. Diabetes Care 35: 2021-2027. 
Citation: Cama VF, Mayola MF, Marí YM, Rivero NA, Ojalvo AG, et al. (2016) Epidermal Growth Factor based Therapy Promotes Intracellular Trafficking and Accumulation of its Receptor in the Nucleus of Fibroblasts from Diabetic Foot Ulcers. J Diabetic Complications Med 1: 111.

Page 8 of 8

3. Berlanga J, Schultz G, Lopez P (2009) Biology of the diabetic wound. Nova Science Publishers.

4. Berlanga-Acosta J, Mendoza-Mari Y, Martinez MD, Valdes-Perez C, Ojalvo AG, et al. (2013) Expression of cell proliferation cycle negative regulators in fibroblasts of an ischemic diabetic foot ulcer. A clinical case report. International wound journal 10: 232-236.

5. Falanga $V(2005)$ Wound healing and its impairment in the diabetic foot. Lancet 366: $1736-1743$.

6. Armstrong DG, Cohen K, Courric S, Bharara M, Marston W (2011) Diabetic foot ulcers and vascular insufficiency: our population has changed, but our methods have not. J Diabetes Sci Technol 5: 1591-1595.

7. Portero-Otín M, Pamplona R, Bellmunt MJ, Ruiz MC, Prat J, et al. (2002) Advanced glycation end product precursors impair epidermal growth factor receptor signaling. Diabetes 51: 1535-1542.

8. Thomson SE, McLennan SV, Twigg SM (2006) Growth factors in diabetic complications. Expert Rev Clin Immunol 2: 403-418.

9. Kasayama S, Ohba Y, Oka T (1989) Epidermal growth factor deficiency associated with diabetes mellitus. Proc Natl Acad Sci U S A 86: 7644-7648.

10. Berlanga-Acosta $J(2011)$ Diabetic lower extremity wounds: the rationale for growth factors-based infiltration treatment. Int Wound J 8: 612-620.

11. Seeger MA, Paller AS (2015) The Roles of Growth Factors in Keratinocyte Migration. Adv Wound Care (New Rochelle) 4: 213-224.

12. Berlanga J, Caballero E, Prats P, López Saura P, Playford RJ (1999) The role of the epidermal growth factor in cell and tissue protection. Med Clin (Barc) 113: 222-229.

13. Berlanga J, Fernández JI, López E, López PA, del Río A, et al. (2013) Heberprot-P: a novel product for treating advanced diabetic foot ulcer. MEDICC Rev 15: 11-15.

14. Berlanga-Acosta J, Gavilondo-Cowley J, López-Saura P, González-López T, Castro-Santana MD, et al. (2009) Epidermal growth factor in clinical practice - a review of its biological actions, clinical indications and safety implications. Int Wound J 6: 331-346.

15. Lopez-Saura PA, Berlanga-Acosta J, Fernandez-Montequin JI, ValenzuelaSilva C, Gonzalez-Diaz O, et al. (2011) Intralesional Human Recombinan Epidermal Growth Factor for the Treatment of Advanced Diabetic Foot Ulcer: From Proof of Concept to Confirmation of the Efficacy and Safety of the Procedure. InTech.

16. Packham S, Lin Y, Zhao Z, Warsito D, Rutishauser D, et al. (2015) The NucleusLocalized Epidermal Growth Factor Receptor Is SUMOylated. Biochemistry 54: 5157-5166.

17. Wang SC, Nakajima Y, Yu YL, Xia W, Chen CT, et al. (2006) Tyrosine phosphorylation controls PCNA function through protein stability. Nat Cell Biol 8: 1359-1368.

18. Mills JL Sr, Conte MS, Armstrong DG, Pomposelli FB, Schanzer A, et al. (2014) The Society for Vascular Surgery Lower Extremity Threatened Limb Classification System: risk stratification based on wound, ischemia, and foot infection (WIfI). J Vasc Surg 59: 220-234.

19. Wagner FW Jr (1981) The dysvascular foot: a system for diagnosis and treatment. Foot Ankle 2: 64-122.

20. Zhan LX, Branco BC, Armstrong DG, Mills JL Sr (2015) The Society for Vascular Surgery lower extremity threatened limb classification system based on Wound, Ischemia, and foot Infection (WIfI) correlates with risk of major amputation and time to wound healing. J Vasc Surg 61: 939-944.

21. Armstrong DG, Lavery LA, Nixon BP, Boulton AJ (2004) It's not what you put on, but what you take off: techniques for debriding and off-loading the diabetic foot wound. Clin Infect Dis 39 Suppl 2: S92-99.

22. Martinez A, Lopez L, Perez R, Anton J, Aguero MM, et al. (1994) Uso del Factor de Crecimiento EpidÃ@rmico humano recombinante en crema de sulfadiacina de plata en el tratamiento de pacientes quemados. BiotecnologÃa Aplicada 11: 209-212.

23. Cinza AM, Quintana M, Lombardero J, Poutou R, PÃ@rez E, et al. (1991) A batch process for production of human Epidermal Growth Factor in yeast. Product characterization. BiotecnologÃa Aplicada 8: 166-173

24. Berlanga AJ, Canan-Haden FL, Chacon CL, Fernandez MJI, Franco PN, et al. (2003) Uso de una composici $\tilde{A}^{3} n$ farmacÃ@utica que contiene factor de crecimiento epidÃ@rmico (egf) para la prevenciÃ ${ }^{3} n$ de la amputaci $\tilde{A}^{3} n$ del pie diabÃ@tico. Google Patents.

25. Schultz GS, Sibbald RG, Falanga V, Ayello EA, Dowsett C, et al. (2003) Wound bed preparation: a systematic approach to wound management. Wound Repair Regen 11 Suppl 1: S1-28.

26. Mayhew TM, Lucocq JM (2008) Developments in cell biology for quantitative immunoelectron microscopy based on thin sections: a review. Histochem Cell Biol 130: 299-313

27. Falcón V, Acosta-Rivero N, Chinea G, Gavilondo J, de la Rosa MC, et al (2003) Ultrastructural evidences of HCV infection in hepatocytes of chronically HCV-infected patients. Biochem Biophys Res Commun 305: 1085-1090.

28. Falcon V, Baranosky N, Castro FO, Montero C, Gonzalez M, et al. (1993) Ultrastructural and immunocytochemical characteristics of hepatocytes from hepatitis B virus infected chimpanzees. Tissue Cell 25: 865-873.

29. Mateo C, Moreno E, Amour K, Lombardero J, Harris W, et al. (1997) Humanization of a mouse monoclonal antibody that blocks the epidermal growth factor receptor: recovery of antagonistic activity. Immunotechnology : an international journal of immunological engineering 3: 71-81.

30. Mayhew TM, Lucocq JM, Griffiths G (2002) Relative labelling index: a nove stereological approach to test for non-random immunogold labelling of organelles and membranes on transmission electron microscopy thin sections. J Microsc 205: 153-164.

31. Buckley A, Davidson JM, Kamerath CD, Wolt TB, Woodward SC (1985) Sustained release of epidermal growth factor accelerates wound repair. Proc Natl Acad Sci U S A 82: 7340-7344

32. Yu FS, Yin J, Xu K, Huang J (2010) Growth factors and corneal epithelial wound healing. Brain Res Bull 81: 229-235.

33. Boulton AJ, Vileikyte L, Ragnarson-Tennvall G, Apelqvist J (2005) The global burden of diabetic foot disease. Lancet 366: 1719-1724.

34. Hatton N, Lintz E, Mahankali M, Henkels KM, Gomez-Cambronero J (2015) Phosphatidic Acid Increases Epidermal Growth Factor Receptor Expression by Stabilizing mRNA Decay and by Inhibiting Lysosomal and Proteasomal Degradation of the Internalized Receptor. Mol Cell Biol 35: 3131-3144.

35. Du Y, Shen J, Hsu JL, Han Z, Hsu MC, et al. (2014) Syntaxin 6-mediated Golg translocation plays an important role in nuclear functions of EGFR through microtubule-dependent trafficking. Oncogene 33: 756-770.

36. van der Pol E, Böing AN, Harrison P, Sturk A, Nieuwland R (2012) Classification, functions, and clinical relevance of extracellular vesicles. Pharmacol Rev 64 676-705.

37. Zhang J, Guan J, Niu X, Hu G, Guo S, et al. (2015) Exosomes released from human induced pluripotent stem cells-derived MSCs facilitate cutaneous wound healing by promoting collagen synthesis and angiogenesis. J Transl Med.

38. Shabbir A, Cox A, Rodriguez-Menocal L, Salgado M, Van Badiavas E (2015) Mesenchymal Stem Cell Exosomes Induce Proliferation and Migration of Normal and Chronic Wound Fibroblasts, and Enhance Angiogenesis In Vitro. Stem cells and development 24: 1635-1647.

39. Demory ML, Boerner JL, Davidson R, Faust W, Miyake T, et al. (2009) Epidermal growth factor receptor translocation to the mitochondria: regulation and effect. J Biol Chem 284: 36592-36604.

40. Yue X, Song W, Zhang W, Chen L, Xi Z, et al. (2008) Mitochondrially localized EGFR is subjected to autophagic regulation and implicated in cell survival. Autophagy 4: 641-649.

41. Lee HH, Wang YN, Hung MC (2015) Non-canonical signaling mode of the epidermal growth factor receptor family. Am J Cancer Res 5: 2944-2958.

42. Lin SY, Makino K, Xia W, Matin A, Wen Y, et al. (2001) Nuclear localization of EGF receptor and its potential new role as a transcription factor. Nat Cell Biol 3: 802-808.

43. Dittmann K, Mayer C, Rodemann HP (2005) Inhibition of radiation-induced EGFR nuclear import by C225 (Cetuximab) suppresses DNA-PK activity. Radiother Oncol 76: 157-161

44. Maga G, Hubscher U (2003) Proliferating cell nuclear antigen (PCNA): a dancer with many partners. J Cell Sci 116: 3051-3060.

45. Bouayad D, Pederzoli-Ribeil M, Mocek J, Candalh C, Arlet JB, et al. (2012) Nuclear-to-cytoplasmic relocalization of the proliferating cell nuclear antigen (PCNA) during differentiation involves a chromosome region maintenance 1 (CRM1)-dependent export and is a prerequisite for PCNA antiapoptotic activity in mature neutrophils. J Biol Chem 287: 33812-33825. 